\title{
Profiling Regional Innovation Ecosystems as Functional Collaborative Systems: The Case of Cambridge
}

\author{
Jukka Viitanen
}

\author{
'( Make no little plans; they have no magic to stir men's blood... ") \\ Make big plans; aim high in hope and work.
}

Daniel H. Burnham (1846-1912)

Architect and urban planner

\begin{abstract}
It has been widely recognized that the national and regional development of innovation ecosystems has been a relatively successful model for regional revitalization, bringing together key actors to perform the relevant technology-driven development processes. The ecosystems have been organized and combine readily public sector interests with private sector business-oriented actions. However, all regions are not uniformly successful, which leaves open the question of how to guide the sub-optimum regional systems closer to the front-runner position. Why do some score better than the others? This article presents both theoretical and practical evidence of global best practice in developing regional innovation hubs and renders a fully integrated innovation hub framework that defines a novel, holistic approach to managing these ecosystems. The framework is tested and validated through a selected case study of Cambridge, United Kingdom, identifying the key ecosystem elements that are necessary for building up a solid foundation for the innovative regions.
\end{abstract}

\section{Introduction}

Changing realities in innovation ecosystems challenge the next generation of development processes for innovation environments at all levels. According to findings in the most recent innovation studies, discussion on closed national innovation systems is rather artificial, while new scientific knowledge and technological inventions are generated in almost purely global settings (Hautamäki, 2008; Ishikura, 2006; Kao, 2007; Saxenian, 2006). The expanding collaboration in value networks brings innovation production closer to the marketplace, the pure technology-push approach is increasingly being complemented with a market-pull type needs analysis for penetrating a growing number of market segments, and a practically free movement of talented people brings down artificial national borders and provides a foundation for a creative transfer of knowledge between the interconnected innovation ecosystems.
Regional innovation ecosystems are the core building blocks for innovation-creation activities from a national innovation policy point of view. They build on a local knowledge base and specialize in bringing out the best in their respective innovation processes. They focus on accumulating academic knowledge and combining it with private sector, market-driven commercialization processes. In most cases, these regional ecosystems are organized around a core-hub organization such as a science or technology park, or alternatively a regional cluster management office, where all the key coordination decisions are made. But, the innovation hub itself is necessarily a much wider concept than only a park or a coordination office. It consists of all the regional innovation ecosystem elements, starting from policies and ending with market-driven business activities.

Accordingly, the work at hand aims at interpreting the systemic interplay of the common characteristics of successful innovation environments and ecosystems in 


\section{Profiling Regional Innovation Ecosystems as Functional Collaborative Systems} Jukka Viitanen

their respective contexts. The article presents both theoretical and practical evidence of global best practice in developing regional innovation hubs and renders a fully integrated innovation hub framework that defines a novel, holistic approach to managing these ecosystems, where all necessary ecosystem elements are being planned coherently under one unified regional master plan to seamlessly connect all framework elements.

The framework is further tested and validated through a selected case study of Cambridge, United Kingdom (UK), which describes Cambridge's internal, inter-domain relations and critical success factors in attracting, keeping, and developing the necessary resources, talent, and capacities for continuous innovation activities. Cambridge's ecosystem profile is analyzed for its capacity and readiness to meet the globalization challenge. It is generally argued that, if and when done properly, the related analyses can reveal a formula for replication and speed up the development of the next generation of environments - not necessarily directly copying and transferring the results "as is" to distant cultural contexts, but more likely imitating the proven functional behaviour for quality results.

One article cannot exhaustively address the complex phenomena at hand. Moreover, some parts of any case analysis are necessarily subjective in interpretation and essentially only represent the authors' own understanding of the core issues and their relational associations. However, it is boldly argued that the combination of the presented framework, case study, and ultimate results provide novel perspectives on the development of successful regional innovation ecosystems for the future. The article gives the reader a chance to familiarize themselves with key concepts related to ecosystem development and the particular characteristics of a global best-practice case site, and then it provides them an opportunity to reflect on the presented notions in relation to their own practices and any specific development and management challenge they are facing.

\section{The Innovation Hub Framework}

Pioneering development of regional innovation hubs has focused to a great extent on the mutually complementing challenges of fostering the local pools of knowhow and orchestrating the actions of the complementing stakeholder groups. It is widely argued that the most attractive regional innovation ecosystems have been built on a strong knowledge base, accumulating a network of complementary innovation processes and ad- vanced combinations of innovation resources (i.e., talent, funding, and infrastructures). The top ecosystems have managed to channel the accumulation of academic knowledge for joint innovation activities and combine the related outcomes with the market-driven commercialization processes.

The triple helix model and knowledge triangle (Etzkowitz, 1997; European Parliament, 2000; Leydesdorff, 2006) approaches have been used to explain these related dynamics and to justify the interlinked relations of the collaborative stakeholder groups. They address the challenges of combining the highly specialized talent pools to productive co-creation processes and utilizing the complementing processes for synergetic outcomes. However, in this article, it is argued that the traditional stakeholder group models and intra-regional analysis will not prepare the regional innovation ecosystems for truly global competition. The future top regional innovation ecosystems will be necessarily embedded in a more globalized, interconnected, and collaborative context, where information, resources, talent, and solutions can flow freely and effectively between mutually complementing or competing locations.

The future challenge for the development of the ecosystems lies in their ability to extend the value network collaboration closer to the marketplace and complement the pure technology-push approach with a market-pull type needs analysis for penetrating a growing number of market segments. A practically free movement of talented people brings down artificial national borders and provides an opening for a creative transfer of knowledge between the interconnected innovation ecosystems. Accordingly, we have to modernize our thinking on future development of regional innovation ecosystems and adopt a more systemic, ecosystem-level approach, which incorporates the triple helix model into a practical ecosystem-orchestration approach.

The following innovation hub framework (LaunonenViitanen, 2011) introduces a comprehensive approach to regional innovation ecosystem development, advocating coordinated planning and implementation of the key ecosystem elements and close interplay among the key innovation actors. The framework guides regional planners, political decision makers, and core-hub organizations to address ecosystem development from a unified cross-sectoral point of view - as a complete regional master planning challenge to connect both public and private sector interests for joint innovation 


\section{Profiling Regional Innovation Ecosystems as Functional Collaborative Systems}

\section{Jukka Viitanen}

actions. If managed properly, these collaborative actions can lead to mutually reinforcing arrangements for parallel innovation processes, and they can facilitate the efficient distribution of best-practice know-how throughout the ecosystem.

The framework illustrates the key regional innovation ecosystem elements that are necessary for building up a successful regional innovation hub (Figure 1). Each element (layer or driver) represents a significant development task of its own, but only in combination can they produce an ecosystem that can truly rise to the globalization challenge and take its place in the value network context. Accordingly, the core management organizations should be able to plan, organize, manage, and further develop the regional ecosystem as a complete set of interconnected elements where interplay and complementarities between the layers give the ecosystem its soul and strength.

\section{Activities driven by the public sector}

The hub framework builds on the national and regional innovation policy foundation, combining actions driven by public policy with private sector interests. The development challenges at the lower part of the pyramid (physical infrastructure and service structure, education policies and curriculum, research and development activities) are addressed primarily from interests driven by public policy, while these elements also have wider societal implications for developing regions and in most cases constitute basic service for the majority of citizens. The infrastructure element addresses issues such as shared R\&D infrastructure (schools, health care centres, shopping malls, etc.), solutions for logistics (road and train networks, airports, etc.), possible park/hub construction, and wider community development projects. The service structure element, in turn, is highly dependent on the actual needs of the ecosystem (not so much on deliberate service provision planning).

The education element should be addressed in a much wider context than the needs of the immediate innovation activity process would otherwise imply, while ecosystem advantages could include quality provision of primary to secondary school (i.e., K-12) education, international multi-lingual high schools, open universities with international degrees, schools for lifelong learning and continuous re-education, and so on. It could also be complementing the $R \& D$ activity element providing high-quality graduate schools for master's

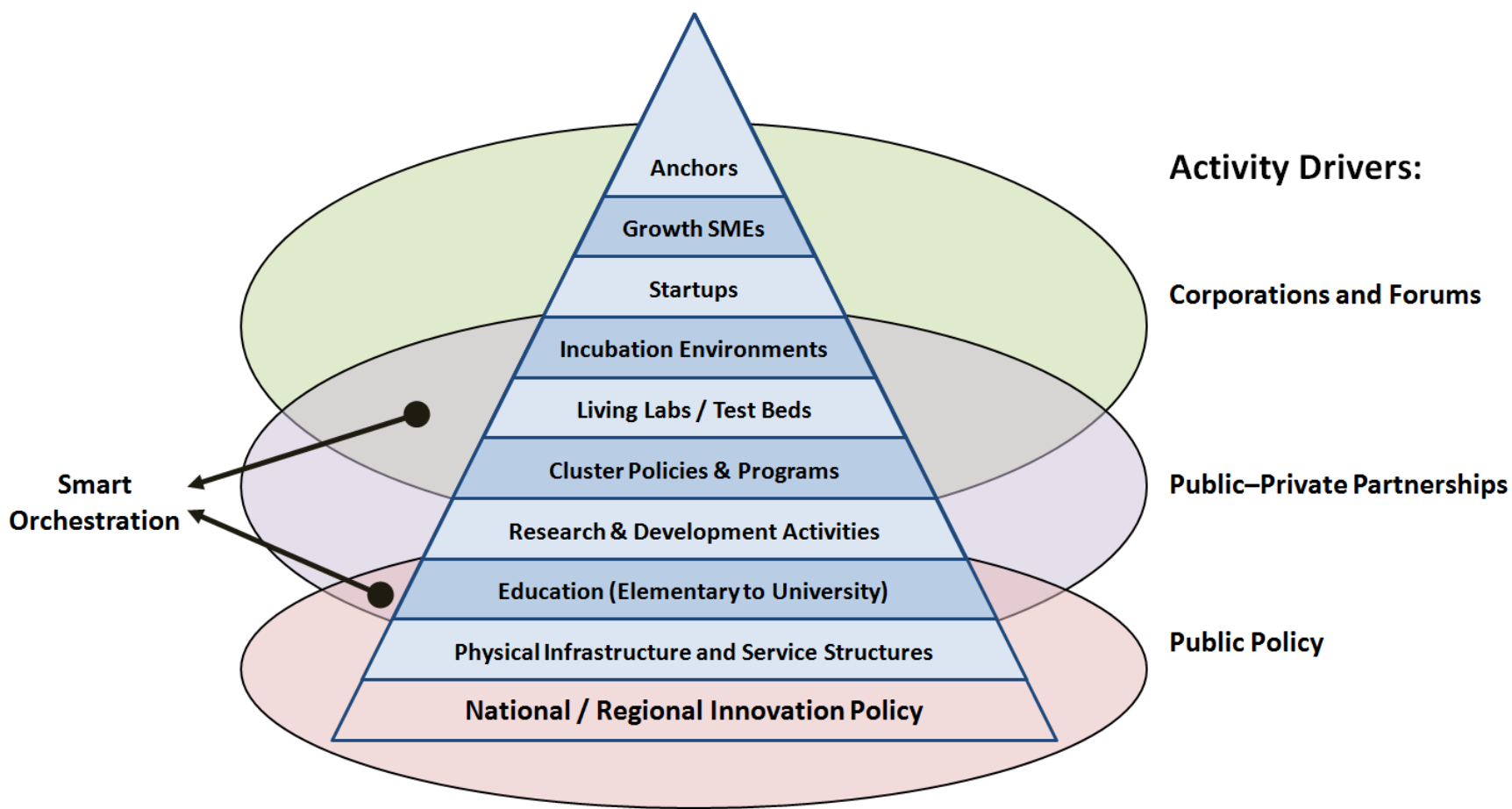

Figure 1. The innovation hub framework and its key regional innovation ecosystem elements 


\section{Profiling Regional Innovation Ecosystems as Functional Collaborative Systems} Jukka Viitanen

and doctoral students, international summer schools for "brain circulation", and executive training programs to refresh management talent.

The R\&D activity element takes account of regional strengths in the research environment and focuses primarily on university-level research. Here, the addressed issues include the quality and breadth of the faculties, unique qualities in research endeavours, output for extended innovation processes, opportunities for multi-disciplinary studies, willingness and ability to participate in contract research projects, technology transfer know-how, and success rates in attracting the centres of excellence to the research units in the region. The university-level research is naturally complemented with close-by national research institutes, which can bring know-how to the innovation hub that is specific to the research sector and attract the private sector to joint development projects.

\section{Activities driven by the private sector}

The innovation processes and collaborative activities towards the top of the pyramid are primarily corporatedriven and forum-driven, where the innovation activities take place much closer to the market and the requirements for speed in creating solutions for the real customers are much higher. The first notion in this context is that every regional innovation hub needs dynamic anchor companies close to the core for their abilities in taking part in global value network competition and for their result-oriented, comprehensive approach in business process development. The anchor companies are usually the key partners in innovation commercialization and have in-house expertise to implement an active intellectual property utilization strategy. However, the "any big company will do" approach is not adequate for identifying the anchor companies, while the business realities and power structure within the companies need to be compared with their innovation-creation abilities. The true anchor companies should have adequate decision-making powers, access to global networks, and concrete innovation creation activities as a relevant starting point for their regional engagement.

The second major group of private actors comprises the high-growth small and medium-sized enterprises (SMEs) and startups. They bring dynamism to the innovation ecosystem with their new ideas and businesscreation drive, which utilize emerging regional knowledge in developing new products and services for the marketplace. The SMEs and venture companies are also major employers within the regions, providing anything from one-third to almost $70 \%$ of all new jobs created, and the high-growth companies perform even better than the average in this respect (e.g., Acs, 2004; Autio, 2005). These SMEs and startups are active users of knowledge-intensive business services (KIBS), outsourcing all non-essential processes (e.g., financing, accounting, legal) to the professional firms and utilizing service providers for contract management, commercialization projects, and channeling necessary funds for growth (e.g., via business angels and venture capitalists). Usually, they also seek partnerships and collaborate actively for subcontracting to boost their reach to markets. Their key role is, ultimately, to provide specialized solutions for the anchor companies and contribute to the value network collaboration (while naturally growing their individual businesses).

\section{Activities in public-private partnerships}

The middle part of the pyramid is a field of shared public-private interest, where innovation activities to support the creation of intellectual property and cross-sectoral collaboration are often planned together. First, the regional cluster programs are aimed at increasing regional innovation and economic activities, encouraging various stakeholder groups to engage in collaborative projects. The programs are used in creating shared vision and values for the region and bringing together cross-sectoral talents at mutually open round tables, which provide risk-free platforms for the exchange of ideas.

The second public-private element introduces living labs and test beds as integrated, collaborative piloting platforms where innovation actors can implement interoperability testing and real-life user experimentation within trusted micro-environments. Typical examples of these platforms include interconnected parts of userdriven cities, real-life experimentation sites on streets, open system platforms for developing mobile applications (with users), and Internet-based, end-user betatesting environments to engage users in an early-stage $\mathrm{R} \& \mathrm{D}$ process.

The final innovation ecosystem element is in the incubation environments, which provide essential, professional growth services for startups and growing SMEs. It should be noted that, unfortunately, some incubation environments have been developed almost purely from a real estate business point of view, where local contractors create a mere physical frame for the incubated companies and market the centres to potential clients 


\section{Profiling Regional Innovation Ecosystems as Functional Collaborative Systems} Jukka Viitanen

with some public-sector backed subvention for rents and basic office service support (phone/mobile/internet connections, secretary services, etc.). In this context, these settings are not considered as incubation centres, but merely as random "office hotels". The real incubation environments are physical locations where a selected group of young companies receive professional support for their management concerns.

\section{The smart orchestration challenge}

The challenge in comprehensive innovation ecosystem planning and management is in combining the parallel interests of the various innovation processes, whether driven by a company or forum, the public sector, or a public-private partnership. As noted, every framework layer is important and the missing parts would be very difficult to be substituted with compensating activities in other layers. Moreover, national and regional innovation policy frameworks put some regulatory limitations on the alternative available paths to be adopted. The related critical management issue can be found in collectively managing the various sectoral interests and interfaces.

It is argued, in this context, that the planning and management of regional innovation ecosystems require special talent and particular abilities to interpret and match the multi-domain interests under one unified management structure. Someone must specialize in aligning the collaborative processes, network relationships, and gradually developing common practices for effective innovation creation, accumulating the required experience, know-how, and connections into one core entity for efficient ecosystem-level coordination. Consequently, orchestrated ecosystem development calls for the establishment of a dedicated hub organization that can take the responsibility for defining a shared vision for the future of the ecosystem, a clear set of objectives for the continuous maintenance of network relationships, and guidelines for effective project coordination and resource allocations throughout the ecosystem. This innovation hub organization can focus its efforts on the ecosystem-level target setting, relationship management, and resource allocations.

In practice, these hub organizations can take over the coordination task of hub planning and management functions, and concentrate their efforts on building up the necessary partnerships for systemic, reciprocal success. They can serve as the ecosystem management offices supporting the innovation actors in their joint activities: i) draft the master plan for the entire ecosystem, ii) build up and complement local networks for quality service provision, and iii) provide hands-on support for intra-ecosystem networking, information exchange, and cross-domain communication. They can orchestrate the joint initiatives and development programs, channel resources to the region and to local innovation actors, and build a positive brand image for the region. In this way, the innovation hub organizations serve others as true "needs-seeds" mediators, value-system matchmakers, and regional networkers.

\section{Turning the Framework into an Analytical Tool}

\section{Emerging needs to understand the systemic nature of regional innovation ecosystems}

It is generally argued that studying and analyzing the competing innovation ecosystems can reveal some common characteristics and universal nominators to explain their success or demise. Scholars and practitioners alike believe that benchmarking and analytical reconstruction of local success models can help them to identify the critical success factors for developing effective, functional, and attractive environments for shared innovation creation. It is possible to study the key building blocks and core management processes of the chosen best-practice environments and then interpret their qualities in given local contexts. If and when done properly, the analysis could reveal a formula for replication and speed up the development of the nextgeneration environments - not necessarily directly copying and transferring the results "as is" to distant cultural contexts, but more like imitating the proven functional behaviour as a common baseline action for quality results. Consequently, the studies go on and quality evaluations are always in high demand.

The investigated phenomena - development processes of regional innovation ecosystems, analyses of the key ecosystem elements, and related management challenges - are all widely researched. Studies on each framework element alone or their direct implications for derived regional ecosystem management could already constitute a complete study of their own (and many highly regarded scholars have chosen to address these questions appropriately). In the same way, a truly comprehensive study of only one regional ecosystem could already serve any scholar in their quest for understanding these complex phenomena (and plenty of published studies of individual ecosystems can be found based on descriptive case study analyses). However, it 


\section{Profiling Regional Innovation Ecosystems as Functional Collaborative Systems}

\section{Jukka Viitanen}

is argued that there is a rising, explicit need to understand regional innovation ecosystems as complete interconnected systems that could and should be managed for "optimized" socio-economic outcomes in a "glocal" context. These generic notions for mutual complementarities, systemic interconnections, and cross-domain management challenges, call for comprehensive ecosystem-level analyses and further development of related models, frameworks, and practical analytical tools.

In innovation ecosystem research, the research problem should focus on studying the key elements and success factors for effective design and management of regional innovation ecosystems: to identify the local strengths in a global context and to discover systemic, ecosystemlevel processes for developing optimized, regional innovation ecosystems. Accordingly, we should deeply investigate global best-practices of regional ecosystem development and extensively analyze the leading innovation regions and their value network connections. On one hand, empirical data and related analyses of global best-practice environments could provide valuable insights into core issues at hand and bring alive the presented innovation hub framework and its local interpretations. On the other hand, the accumulating insight would make it possible to test, interpret, and complement (if necessary) the frameworks already used, and develop them further for generic global use.

\section{Case study outline and operationalization of the framework}

The regional innovation ecosystem case studies should be conducted in compliance with qualitative research approaches and multiple case study methods to actually address and grasp the complex nature of the investigated phenomenon. The case design and conduct should be decided as follows. The investigators should visit all the sites for the actual case study data collection, interview the local key actors, and observe the present state of the developed ecosystems (infrastructure, service structures, availability of cross-sectoral facilitation, etc.). The empirical data collection must be conducted using multiple sources of evidence (e.g., written reports, archival records, previous studies, public information) to find converging evidence to crosscheck the findings. The interviews are recommended to be conducted using a semi-structured interviewing technique where the interviewers are following a predetermined set of questions, but allowing the interviewees to respond in a relaxed, conversational manner, jumping around freely from topic to topic in their expression of ideas. The applied technique allows collection of large amounts of data quickly and efficiently, enabling exploration of many topics in a relatively short time. The immediate follow-up questions make it possible clarify and interpret the key concepts.

The developed innovation hub framework (with its firm theoretical foundations and advanced extensions) is an appropriate tool to analyze regional innovation ecosystems. The framework thoroughly describes the core elements (layers) needed for systemic ecosystem development and defines their key characteristics in advancing the globalization of ecosystems. It guides and frames the related discussions of regional innovation policies, collaborative actions for joint creation of intellectual property, and coordination of the local management processes. Consequently, the framework seems to meet the criteria for extensive use in exploratory case studies.

However, it is evident that the framework needs to be operationalized further for analytical purposes. In this study, it has been operationalized as follows. First, it was decided that each layer must be explicitly addressed in the analysis to maintain the uniformity of cases regardless of the rating, level, or qualities in given contexts (sites) of individual layers. This approach leads to a candid ecosystem-level analysis and treats each case equally on an aggregate level. Second, it was relevant to determine in advance the key criteria for layer-bylayer analysis to guide the basic case design, the selection of appropriate methods for data collection, and the ultimate way to use the chosen framework in the analysis. It was important to keep the focus on the chosen research questions at all times and maintain an unchanged case setting and approach for the case study site.

The layer-by-layer criteria for analysis were determined by sets of key questions for each layer (Table 1). The questions were chosen to best characterize the developmental state and potential of each layer in terms of relative preparedness for "glocal" value system contributions, openness for cross-sectoral collaborative activities and actions, and resident abilities to engage and succeed in global, inter-ecosystem competition. Consequently, it was never enough to just claim and state that some elements exist in the local ecosystem, but to broaden the analysis to include evaluations of the scale, scope, and breadth of use of each element. 


\section{Profiling Regional Innovation Ecosystems as Functional Collaborative Systems} Jukka Viitanen

Table 1. Key questions for the layer-by-layer analyses and evaluations

\begin{tabular}{|c|c|}
\hline Element & Questions \\
\hline Anchor Companies & $\begin{array}{l}\text { - How many domestic/global anchors are located within the ecosystem? } \\
\text { - How active are these anchors in cross-sectoral collaboration? } \\
\text { - Have they had an interest in managing the "glocal" activities? } \\
\text { - Do they engage actively in open innovation processes? }\end{array}$ \\
\hline $\begin{array}{l}\text { Startups \& Growth- } \\
\text { Oriented SMEs }\end{array}$ & $\begin{array}{l}\text { - How many startups and ventures are born within the ecosystem? } \\
\text { - How growth-oriented have the local SMEs been (going public)? } \\
\text { - How internationalized (global) is the venture habitat? } \\
\text { - Do the ventures and SMEs participate in "glocal" joint activities?' }\end{array}$ \\
\hline Incubation Environments & $\begin{array}{l}\text { - What kind of incubation services are available to tenants? } \\
\text { - How well does the incubation system facilitate SME growth? } \\
\text { - How open is the incubation system for global activities? } \\
\text { - Do the incubators participate in "glocal" joint actions? }\end{array}$ \\
\hline Living Labs \& Test Beds & $\begin{array}{l}\text { - What kinds of test environments exist within the ecosystem? } \\
\text { - Are testing facilities and systems open to all actors? } \\
\text { - How well are the test environments connected to the outside? } \\
\text { - Do the test environments involve end users in joint activities? }\end{array}$ \\
\hline $\begin{array}{l}\text { Cluster Policies \& } \\
\text { Programs }\end{array}$ & $\begin{array}{l}\text { - How many cross-sectoral cluster programs are established? } \\
\text { - Have these programs been able to induce true collaboration? } \\
\text { - How well do they run after the initial public support runs out? } \\
\text { - Are these programs connected to the outside (globally)? }\end{array}$ \\
\hline R\&D Activities & $\begin{array}{l}\text { - How many top-quality actors are located within the ecosystem? } \\
\text { - How active are these actors in cross-sectoral collaboration? } \\
\text { - How successful has the R\&D system been in a global context? } \\
\text { - How open are the R\&D systems for global collaboration? }\end{array}$ \\
\hline $\begin{array}{l}\text { Education (Elementary to } \\
\text { University) }\end{array}$ & $\begin{array}{l}\text { - How well has the education system developed (at all levels)? } \\
\text { - How well does the education system support continuous development of human resource skills? } \\
\text { - Are there any truly international actors located within the ecosystem? } \\
\text { - How well does the education system meet global criteria? }\end{array}$ \\
\hline $\begin{array}{l}\text { Infrastructure/Service } \\
\text { Structures }\end{array}$ & $\begin{array}{l}\text { - How well does the local infrastructure/service structure meet global criteria? } \\
\text { - Are the local structures truly connected to national infrastructure? } \\
\text { - Is the local infrastructure truly connected to the world (logistics)? } \\
\text { - Are all the services readily available in English for foreigners? }\end{array}$ \\
\hline $\begin{array}{l}\text { Innovation Policy } \\
\text { Framework }\end{array}$ & $\begin{array}{l}\text { - How well have the local actors managed to link in and benefit from the national policy } \\
\text { framework, programs, and structures? } \\
\text { - How successful have these actors been in attracting funding? } \\
\text { - How well do the framework conditions/incentive structures meet needs? }\end{array}$ \\
\hline
\end{tabular}




\section{Profiling Regional Innovation Ecosystems as Functional Collaborative Systems} Jukka Viitanen

In other words, the related analysis had to comprise evaluations on the actual relevance, functionalities, and impact of organized activities within the given layer. The individual outcomes were recorded and then compared against an ideal, fully-developed, best-practice layer - case-by-case, layer-by-layer, - and given a numerical value (percentage $0-100$ ) that indicated their levels of maturity in global ecosystem comparisons (the higher a numerical value, the more advanced an individual layer would appear to be). The use of numerical values was chosen to give each layer a concrete measure to illustrate its present state and potential, and to make it easier to summarize the findings in a powerful and concrete way.

The individual layer analyses were, finally, matched against the whole innovation hub framework to draw a comprehensive ecosystem profile (case-by-case), which would approximate the overall ecosystem maturity and highlight the key gaps in the present state of the ecosystem in question. The profile summarizes the systemic analysis and provides a graphical representation of the case study outcome in question. In some cases, these profiles can even reveal the underlying characteristics of local development strategies and guide the next generation of ecosystem planners in their efforts to identify the right path to the future. It is argued, therefore, that the ecosystem profiles can be used effectively in comparing the best-practice environments and identifying the local development needs for systemic actions.

Each case study was summarized in the end using a combination of three parallel continuums, which collectively quantify each ecosystem's capacity and readiness for meeting the globalization challenge (Figure 2). It is argued that the future success of a regional ecosystem is strongly related to its ability to link local strengths to emerging global opportunities, and to its readiness to engage in truly global value network collab- oration with other first-class environments. The best ecosystems could serve as interconnected innovationcreation and market-entry hubs, providing a platform for market-specific product and service localizations as well as a route to local markets. Accordingly, it was of utmost importance to estimate and assess the capabilities in related terms.

The first continuum assesses the level of reach and impact of the individual ecosystems in their respective countries and regions. It is argued that all ecosystems could be considered relevant within their local context, but only some can advance to meet the national or global benchmarks and criteria. However, all ecosystems could benefit from shifts to higher grades and, consequently, should aim for the furthest possible reach.

The second continuum appraises the advancement of the ecosystems as global hubs. Some of them are still on the starting line and have only begun to address the globalization challenge while others have thoroughly advanced mechanisms and processes in place for fullscale global engagements. It is argued that the future "winners" must shift to the right and actively seek a position as a global hub.

The third continuum assesses the overall maturity of the ecosystems' hub-management processes and structures. Some ecosystems have been developed following a systematic master plan (drafted by regional or national authorities) with good results while others have taken an almost pure, organic route to excellence. Regardless, it has become evident (through analyses) that all ecosystem-development processes have enjoyed some degree of coordination, which has resulted in broader cross-sectoral engagements, in-depth value-network development processes, and advanced innovation-creation practice. Consequently, hub management pro-

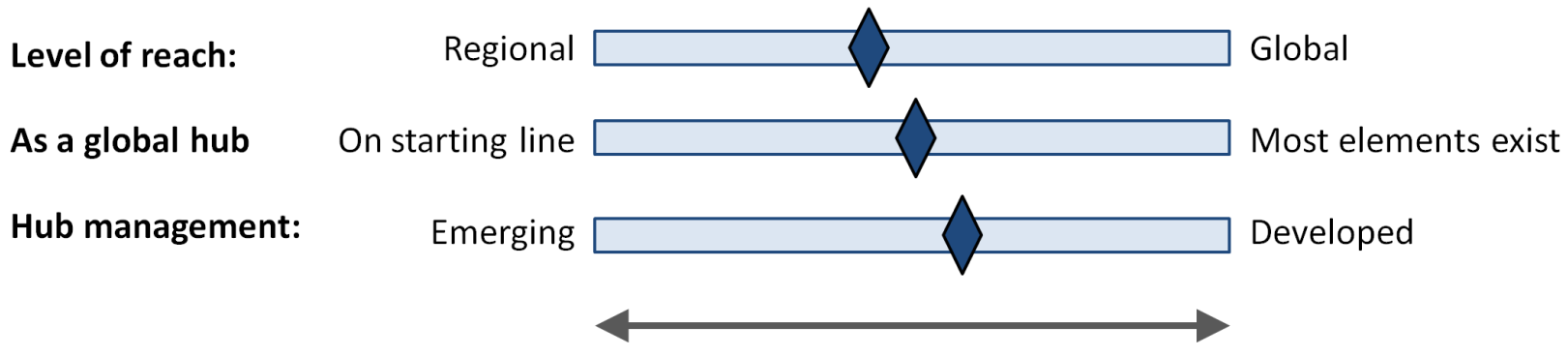

Figure 2. An example of a continuum combination 


\section{Profiling Regional Innovation Ecosystems as Functional Collaborative Systems} Jukka Viitanen

cesses are the core elements in building up the innovation capacity of the individual ecosystems and their ability to engage in global value-network competition. Again, shifts to the right of the continuum improve the overall position of the ecosystems.

\section{Approach to data collection}

The case design and conduct followed the recommendations described above. The author visited the selected site for the actual data collection, interviewed the local key actors, and observed the present state of the developed ecosystems.

The case data was collected between April 2009 and August 2010. The collected data was archived in a research database and rearranged to meet the theoretical framework and presentations, facilitating the further analyses and possible cross-site comparisons. The unit of analysis was determined at the regional innovation ecosystem level with embedded collection and analysis of data from both sub-unit (framework layer) and aggregate (systemic ecosystem level) levels. The results of the study are presented as follows.

\section{Case Study: Cambridge}

The Greater Cambridge area (referred to in this context as the Cambridge Sub-Region) is located in close proximity to London, the UK's capital and largest city, thereby benefiting to a great extent from its advanced service structures and readily available resource pools. Cambridge is world-renowned for its academic traditions, the scientific pre-eminence of the University of Cambridge, and the high standard of living as a beautiful countryside community. The City and the University are located right in the heart of the sub-regional ecosystem, interconnecting a number of local communities, innovation platforms, and research institutions into a mutually reinforcing structure of regional development (Granger, 2009). It is argued that the Cambridge Sub-Region enjoys a true critical mass of businesses and academic institutions involved in high-tech $\mathrm{R} \& \mathrm{D}$ and technology commercialization, creating a rich ecosystem for targeted innovation creation, timely technology transfers, and a dynamic "engine of economic growth" in the broader regional and national contexts.

The Cambridge Sub-Region has turned in impressive innovation-related performance across a broad range of sectors over the last four decades. It has been recognized as a key contributor to the UK economy, which can be seen directly in national GDP figures and indirectly through a range of productivity gains throughout the economy - including the ability to attract worldclass $\mathrm{R} \& \mathrm{D}$ facilities to the sub-region, transferring ideas and knowledge to other parts of the economy, and advancing the growth of many highly entrepreneurial companies (GCP, 2008). The sub-region has enjoyed steady growth in employment in knowledge-based professions and its skilled workforce is seen as one of the biggest regional assets in terms of global competitiveness.

Cambridge and its surroundings are sometimes referred to as Silicon Fen (tinyurl.com/glh6bma), an allusion to Silicon Valley (tinyurl.com/roc7a), because of the density and qualities of technology incubators and hightech businesses that have developed in and around the 11 science and technology parks circling the city. The business landscape has a diverse blend of university and corporate spin-offs, growth-oriented SMEs, and a strong presence of publicly listed companies, which are professionally supported by numerous technology consultancies and other business service providers. Consequently, the Cambridge Sub-Region has become a preferred destination and target for angel, venture capital, and foreign direct investments from all over the world, and it has become one of the top innovation regions in Europe in terms of total institutional investment per capita (Library House, 2008).

It should be noted in this context that the cited development over past decades and the regional collaborative structures that emerged are the ultimate outcomes of a rather random "bottom-up" development of gradually aggregating, innovative technologies and companies, embedded in a rich and diverse science base of worldclass universities and a readily available, abundant human resource pool of top-quality talent and minds. It is argued that this so-called "Cambridge Phenomenon" emerged from a myriad of local factors and individual decisions (not as a result of a deliberate plan) over a longer period of time and almost as a gradually self-fulfilling prophecy, with no managed, intervening local processes. The local dynamism has grown from the collective and cumulative contributions of passionate individuals and forward-looking organizations alike, acting (perhaps) in their self-interest but understanding the strategic importance of shared vision, purpose, and coordinated actions. Their actions have resulted in advanced structures of region-wide networking, bringing together diverse interest groups to guard and reinforce the shared processes, enabling them to partner for 


\section{Profiling Regional Innovation Ecosystems as Functional Collaborative Systems}

\section{Jukka Viitanen}

timely transfers of information and technologies for effective commercialization and joint development of local communities. This strong culture for mutually reinforcing interaction has translated readily into local economic dynamism and regional innovation excellence.

\section{Key figures and facilities}

The Cambridge Sub-Region refers to an area within a 40-kilometer radius around the City of Cambridge with a resident population of 750,000 and a working population of 360,000 people. It hosts a total of 27,500 businesses employing over 43,000 high-tech professionals and generating an annual regional GDP of about $£ 15.8$ billion GBP ( $\$ 26.6$ billion CAD). According to recent estimates, the region has five times more $R \& D$-related jobs than the national average and about $46 \%$ of the local workforce is educated to a university degree level (GCP, 2008; Granger, 2009).

The sub-region receives $17 \%$ of the national public R\&D expenditure and $9 \%$ of total university research funding (EEDA, 2009). The local ecosystem hosts two major universities, the University of Cambridge ranked 4th overall in the World University Rankings 2016-2017 (Times Higher Education, 2016) - and Anglia Ruskin University, which educate a combined student population of 49,000 in all fields of science. The University of Cambridge alone has 31 colleges, 3 graduate colleges, and 150 departments, faculties, and schools to provide the highest-quality university-level education and researcher training in the country. It also boasts 150,000 alumni graduates worldwide and extends its reach to all continents through its extensive network of collaborative institutions. Over $70 \%$ of all published research work and results from the University of Cambridge are rated as either world-leading or internationally excellent, positioning it second in overall UK university rankings (EEDA, 2009; University of Cambridge Web, 2010). Anglia Ruskin University complements the local university scene by offering mostly undergraduate-level education and degrees for both onsite and offsite student populations. Nine percent of its almost 40,000 students come from outside the European Union (CUG, 2016).

By 2008, there were, in total, 1,500 high-tech companies in the Cambridge Sub-Region (half of which engaged directly in R\&D while the other half focused on supporting the first half through advanced service provision), most of which were located in or around one of the 11 regional science parks. The ecosystem hosts several
$R \& D$ centres of globally-renowned industrial giants (e.g., Toshiba, Microsoft, Nokia, ECM, ARM, Qualcomm, Philips, Takeda, AstraZeneca, Pfizer), which participate actively in horizontal collaboration and seek regional partnerships with first-class research teams and emerging high-tech ventures. Local businesses in the sub-region spend more on R\&D and make more innovation investments than actors in any other region in the UK (EEDA, 2009). The local industries, startups, and ventures have been able to attract over $£ 200$ million GBP (\$340 million CAD) in venture funding to the ecosystem. Spin-outs from the University of Cambridge alone have received more venture capital investment than those of any other UK university, testifying to the high qualities of both research and innovativeness in commercializing potential ideas for actual use in the marketplace. With these numbers, the Cambridge SubRegion accounts for $7.8 \%$ of all UK venture capital investments and ranks first in Europe in terms of investments per capita, contributing to the total of almost $£ 800$ million GBP ( $\$ 1.3$ billion CAD) of institutional capital currently committed into the cluster (Greater Cambridge, 2009; Library House, 2008).

\section{Key players in the innovation ecosystem}

The Cambridge Sub-Region features four groups of key players in its innovation ecosystem:

\section{Public sector actors and park management}

\section{Universities and national research institutes}

\section{Private industry and incubators}

\section{Collaborative networks (official and social)}

The key public sector actors, the Cambridgeshire County Council and five District Councils, have continued to support the local ecosystem development for decades. They have lobbied for national funding and program support for the sub-region, have actively promoted the local triple helix collaboration, and have engaged public actors (councils, agencies, and working groups) in joint innovation platform development. These local authorities have been keen supporters of various forms of public-private partnerships and, consequently, put special emphasis on developing specialized expert organizations to facilitate these processes. Accordingly, part of the public funding is channeled into "softer", coordinating infrastructure/service structure maintenance. 


\section{Profiling Regional Innovation Ecosystems as Functional Collaborative Systems} Jukka Viitanen

The Greater Cambridge Partnership (GCP) was established to coordinate the regional public sector activities and related initiatives for public-private-third-sector partnerships. It plays an important role in direct program implementation, securing funding for key economic interventions and lobbying for government support to achieve regional visions and development objectives. Moreover, it orchestrates cross-domain collaboration, information exchange, and joint regional programs for all involved, and it liaises with other public agencies and expert organizations. Cambridgeshire Horizons, in turn, coordinates all the regional infrastructure and service structure development initiatives to maintain local functionality and dynamisms around innovation activities. It promotes design of the highest quality and channels information, funding, and project flows in and between the local districts and communities (Cambridgeshire Horizons, 2008).

As mentioned, the Cambridge innovation ecosystem hosts 11 science and technology parks. Each park has its own management structure and is run as an independent, for-profit organization that has to find its place in the broader ecosystem context against the severest economic competition. This setup ensures that each established structure contributes, in practice, to the common good, and strengthens the local ecosystem in a meaningful way.

As argued earlier, the private industry structure has matured gradually over time and today plays its fully functional role in the sub-regional economy. The ecosystem hosts several $R \& D$ centres of globally-renowned industrial giants and dominant market leaders in their respective industries. It has been noted in several contexts (EEDA, 2009; Library House, 2008) that these true anchor companies are exceptionally open and ready for horizontal collaboration in this particular, regional context, and that they engage actively in local networking, joint research and development and innovation (R\&D\&I) processes, and commercialization of emerging technologies and solutions. They take shared responsibility for developing the local infrastructures, programs, and interaction (for all to benefit), and they engage in open dialogue on public forums, commit their resources (time and money) in collaboration, and support the overall wellbeing of the entire sub-region as their key asset for future success.

These activities of anchor companies are conveniently complemented with a network of hundreds of hightech startups, growth-oriented SMEs, and technology providers, which operate in closely interlinked, industry-based value systems within the ecosystem (usually in or around one of the local campuses or parks). Strategic partnerships with leading anchors serve the venture habitat in identifying the best commercialization and market opportunities at the right time, and in building up the necessary value networks for rapid business ramp-ups. The strong technology clusters have attracted both professional service providers and investors into the sub-region and, today, the Cambridge innovation ecosystem hosts a vibrant group of technology consultancies, venture capital companies, business angels, and knowledge-intensive business service (KIBS) providers. These actors contribute, for their part, to the development of a new generation of entrepreneurs, ecosystem-level learning processes and smooth transfer of knowledge throughout local value networks.

There are several incubation centres within the ecosystem, but St John's Innovation Centre is considered the leading provider of comprehensive incubation support services in the sub-region. It provides premises, offices, and technical and business development services to its clients in both early and growth stages. The Innovation Centre has developed and implements an internationally recognized model for incubation, collaborates with nine European incubation partners, and supports all the main regional programs (Business Link, Enterprise Hub, Cambridge Corporate Gateway, etc.) for entrepreneurial development (St John's Innovation Centre, 2010). It is commonly argued that the Innovation Centre provides a dynamic support structure to accelerate the growth of ambitious innovative firms in the Cambridge Sub-Region. Apart from this "pure" incubation, the Cambridge innovation ecosystem is known for its numerous support structures for entrepreneurial activities. The Judge Business School, Cambridge Enterprise, Cambridge-MIT Institute, and the Centre for Entrepreneurial Learning all support the active development of the local venture habitat.

The entire ecosystem is built up and around its core university, the University of Cambridge, which is home to over 18,000 full-time students (one-third enrolled in graduate programs) and more than 12,000 scholars and staff. Fifteen percent of the undergraduate students, almost fifty percent of the postgraduate students, and thirty percent of the scholars/staff originate from outside the UK. Therefore, even though the University is a publicly funded institution committed to the education of British students, it is also seen as an open platform 


\section{Profiling Regional Innovation Ecosystems as Functional Collaborative Systems}

\section{Jukka Viitanen}

for education and research excellence for people from around the world, who thirst for knowledge and show exceptional promise and a capacity for first-class, innovative thinking (University of Cambridge Web, 2010). In addition, the University of Cambridge and the mostly undergraduate Anglia Ruskin University, the Open University in the East of England opens up the UK education system to distance and adult education populations by providing undergraduate part-time education to over 17,000 students in the sub-region.

The Cambridge Sub-Region also hosts dozens of national research institutes as one of the key research platforms of the UK and most of them can be found within the University of Cambridge, next to the key faculties and research teams in different disciplines. For example, most of the (bio)medical institutions are located at the same site as the School of Clinical Medicine (such as Cancer Research UK, the Laboratory for Molecular Biology, Brain Research and Repair Centres, and the Institute for Public Health) at Addenbrooke's Biomedical campus and are supported by the Medical Research Council of the UK. These collaborative, co-location arrangements give all researchers direct access to the accumulated scientific knowledge and efficiently bring together the brightest minds to work on interrelated, multidisciplinary research themes and topics.

The local universities and research institutes are very keen supporters of academia-industry collaboration and entrepreneurial activities (Barrell, 2005). The university organizations participate actively in local (research) infrastructure development and help the private sector to utilize and commercialize the created intellectual properties in their everyday businesses. There are currently 16 different groups within the University of Cambridge alone, supporting entrepreneurial activities (venture creation, technology transfer, licensing, networking, etc.) and local innovation processes in specific areas of research. For example, the society for Cambridge University Entrepreneurs (CUE) organizes a range of co-learning events, including lecture series and pitching challenges. Between 1999 and 2009, it has received over 450 entries to its competitions and awarded grants of around half a million dollars USD to over 40 business ideas. These ideas have turned into real companies that have managed to raise a further $£ 40$ million GBP (\$66 million CAD) in investments (Granger, 2009).

The scattering of the sub-regional innovation infrastructures around the City of Cambridge, in numerous campuses and park environments, makes it difficult (at times) to communicate effectively across domains.
Therefore, the local ecosystem needs to be collectively engineered for horizontal collaboration - to bring together otherwise distant actors on joint platforms. Consequently, the local actors have become very active in networking and building up virtual communities to fight the risk of isolation.

The ecosystem hosts a wide variety of networking support organizations that provide solid foundations for intense interaction among the local actors. Local Chambers of Commerce together with Cambridge Enterprise \& Technology Club, Cambridge High-tech Association of Small Enterprises, and the Cambridge Network, provide dynamic collaborative platforms for active interchanges and bring together expert and special interest groups to facilitate mutually beneficial exchanges of ideas, technologies, and solutions. The Cambridge Network has a membership of about 1,400 like-minded people from business and academia that link to each other and to the global high-tech community. These activities offer access to scale economies on the local training scene and improve the overall quality of training/coaching/mentoring within the peer networks. As a testament to event qualities, participating industries fund almost all the activities of the network.

One Nucleus, a not for profit membership organization representing life science companies was formed in 2010 as a merger of the Eastern Region Biotech Initiative (ERBI) and the London Biotechnology Network (LBN). The organization brings together actors within a given industrial context and promotes horizontal collaboration throughout the local value system. It organizes annual events and monthly meetings, which usually lead to concrete partnering exchanges, boosting local efficiencies and concrete business creation. Today, it has over 500 members and is recognized as Europe's most successful regional cluster group.

\section{Analysis of the Co-existence of Ecosystem Elements in the Cambridge Sub-Region}

The Cambridge Sub-Region can be characterized as a fully-functional innovation ecosystem for coordinated knowledge creation, efficient transfer of technology and orchestrated high-tech commercialization, as well as a dynamic "engine of economic growth" in the broader regional and national contexts. It is seen as a true "hotbed" of advanced startup and venture development activities, bringing together academia, established businesses, and industrial clusters to commercially exploit scientific knowledge in proprietary or open innovation creation processes. As such, it 


\section{Profiling Regional Innovation Ecosystems as Functional Collaborative Systems} Jukka Viitanen

provides both an attractive setting and an advanced collaborative culture for strategic contract research and targeted innovation-creation activities across complementary domains.

The local actors are thoroughly interconnected through advanced partnerships and promote collective learning and networking within regional value systems. As a consequence, organizations and individuals are very keen and willing to help each other, which is reflected in the high level of engagement of the business community in collective innovation activities throughout the sub-region (Granger, 2009). The compact size and relatively small local population indirectly support the emergence of truly tangible horizontal mechanisms and effectively interconnect the core actors for shared program/project development.

However, the sub-region has started to hit its limits of indigenous growth due to gradually degrading regional conditions (e.g., high housing prices, infrastructure deficits, skills shortages, slowing growth of ventures and startups, stretched essential services) and, consequently, the long-term health of the local ecosystem may be at risk (Greater Cambridge, 2009).

Some argue that, although the sub-region and its innovative clusters are significant in regional and national contexts in certain measures, the ecosystem, as a whole, remains relatively small when compared with other leading global clusters. The sub-regional ecosystem lacks the necessary critical mass as a single entity to fully compete on a global scale (Granger, 2009; Library House, 2007). Consequently, it may be time for both regional and national decision makers to join forces to develop a common vision for the Cambridge's future and secure the necessary resources to build on the set foundations of shared core strategies, dense agglomeration of quality actors, open collaborative culture, and world-class research excellence.

Overall, the innovation ecosystem profile of the Cambridge Sub-Region is better balanced than many of its global peer innovation hubs, as shown in Figure 3, where a numerical value (percentage 0-100) indicates each layer's level of maturity in global ecosystem comparisons (i.e., the higher a numerical value, the more advanced an individual layer would appear to be). The regional and national governments are well aware of the importance of the ecosystem in terms of both innovation creation and economic impact. Consequently, the local councils have granted generous support to the local actors and developers. They have lobbied for national funding and program support for the sub-region, have actively promoted the local triple helix collaboration, and have engaged public actors (councils, agencies, and working groups) in joint innovation platform development. The regional strategies have been drafted in an open dialogue between key actors and resulted in a shared vision for the future. Accordingly, the policy makers are committed to joint actions and readily assign the necessary resources (time, human resources, and funding) for supporting cross-domain innovation activities.

The local infrastructure for innovation-related activities is extremely well-developed, with one actor a worldrenowned top-tier university, dozens of national research institutes, and a more than an adequate number of facilitative horizontal structures in place. Moreover, the ecosystem hosts a total of 11 science and technology parks, numerous incubation environments, and dozens of private R\&D units and facilities, which readily combine their innovation resources and dedicated infrastructures for open, collaborative processes. The basic infrastructure in the Cambridge Sub-Region is in good order, providing fully-developed structures for all to share and connecting the ecosystem to the Greater South East "supercluster" involving London and Oxford. The regional service structures are equally well-developed, providing a full set of basic KIBS, incubation, and globalization services complemented with advanced investment schemes for meeting the diverse funding needs.

However, as noted, the Cambridge Sub-Region faces serious challenges in its future infrastructure development. As the region has been developing over hundreds of years in the middle of an idyllic countryside setting, the existing road and train networks are rather underdeveloped to meet the rapidly increasing population and their commuting needs. The local roads are often congested and intra-ecosystem commuting is both time-consuming and frustrating, limiting the possibilities for continuous dynamic interchanges. Moreover, affordable housing has become a scarce resource with a booming real estate market, and the existing dwellings rarely meet the most modern requirements for symbiotic functionality and comfort. It is evident that the local infrastructure development has not kept pace with changing ecosystem realities and may also begin to limit the overall innovation potential. Under these circumstances, dispersed innovation infrastructures cannot be in optimal use until the ease of movement and effective interconnections between the complementing clusters of innovation are restored. 


\section{Profiling Regional Innovation Ecosystems as Functional Collaborative Systems}

\section{Jukka Viitanen}
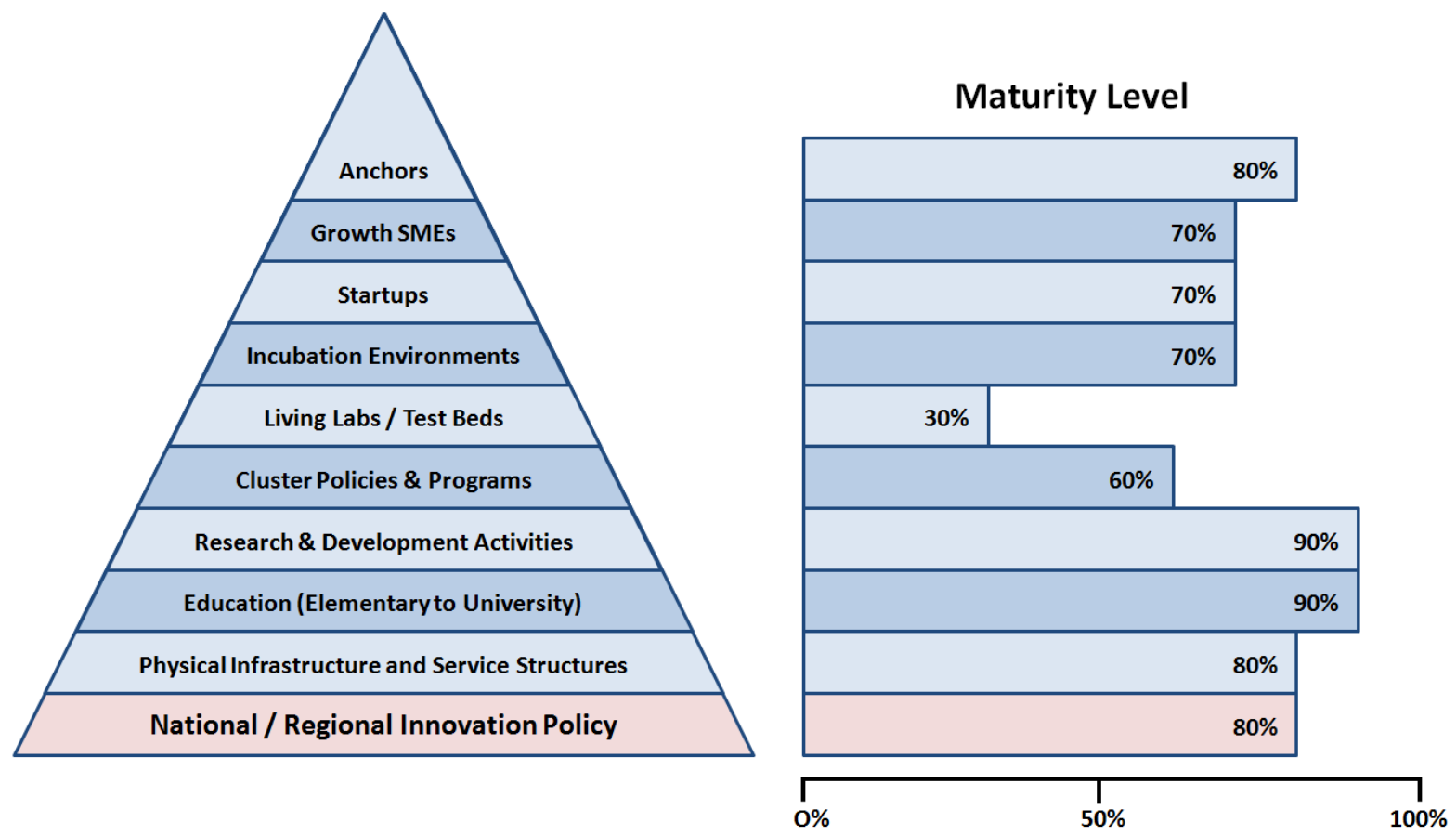

Figure 3. The ecosystem profile of the Cambridge Sub-Region and the maturity levels (\%) for each of its layers

The regional education system meets well the national and global standards for the highest-quality primary to secondary (i.e., $\mathrm{K}-12$ ), undergraduate, and graduate curricula, offering pupils, students, and researchers alike an attractive choice of location. The University of Cambridge has participated actively in developing local education environments and supported all educators in their efforts to meet the truly global demand. Consequently, both public and private schools in the area enjoy a good reputation and continuously develop their curricula for domestic and international students. Moreover, a significant number and range of vocational training institutions, language schools, and further-education centres provide advanced skills-development courses for various student segments. They complement the educational structures at the university level and maintain, on their behalf, the skill profiles and innovation capacity of resident asset pools. In addition, they collaborate with local intermediary organizations (societies, associations, and agencies) on a human resources re-training scene and promote continuous life-long learning among regional stakeholder groups (GCP, 2008).

The Cambridge Sub-Region is world-renowned for its academic traditions, the scientific pre-eminence of the University of Cambridge and first-class research infrastructures. According to recent studies, the sub-region ranks as a high performer among UK regions in overall research quality, public R\&D investments, and business involvement in all innovation activities, and performs on a truly global level in various fields of science and research in terms of originality, significance, and rigour (EEDA, 2009). The University of Cambridge is a major recipient of governmental R\&D support for maintaining and upgrading continuously its research infrastructures and educational facilities, offering the sub-region the necessary foundation (and resources) for continuous science and technology and $R \& D$ collaboration. Its diverse science base and multidisciplinary research capabilities have fostered the ability and capacity to diffuse knowledge and experience through the regional value systems.

R\&D activities are also performed at Anglia Ruskin University, resident science parks, and dozens of public and private research institutes. In combination, they offer open, fully-functional platforms and research environments for gradually converging high-tech clusters of information technologies, biotechnologies, and nanotechnologies. They also breed an emergence of practical, application-oriented research culture bringing into focus the cross-domain settings for collaboration and connecting academic problem-solving excellence to practical industry-specific applications. 


\section{Profiling Regional Innovation Ecosystems as Functional Collaborative Systems} Jukka Viitanen

The first-class R\&D infrastructure and platform are the necessary building blocks for initiating local innovation processes and attracting global talent to the ecosystem to strengthen the local knowledge and asset pools. In the Cambridge Sub-Region, the regional research and development and innovation (R\&D\&I) collaboration has decades-long traditions and has been translated into functional, knowledge-based cluster development processes, improving collective capacities to innovate on a grand scale. Consequently, the local private sector actors in Cambridge are ranked, today, as the leading high-performers in the UK in new product/process introductions, and lead their peers in overall high-tech patenting performance (EEDA, 2009).

The innovation culture at the Cambridge Sub-Region embraces regional cluster development. Local collaboration is built around a regional Enterprise Hub Strategy, which aims at developing the mechanisms to boost industry-specific networking and collaboration. The specific actions include shared, long-term vision work and program planning for entire high-tech clusters and industry groups, and attraction of both public and private funding support to boost cross-domain innovation activities. The emerged partnerships have matured over time and brought together the key decision makers to draft broader socio-economic strategies for the extended sub-region, to support local community development, and to coordinate the more generic sub-regional plans for continuous growth (in numbers of homes, jobs, and available amenities).

However, the resident science parks within the ecosystem are not very keen, or active, participants in developing the local cluster structures. Given that they operate under strict financial control as for-profit organizations, they execute an almost pure real estate business model, limiting open possibilities for complementary service development, which would be essential for creating high-potential new businesses and local programs for joint cluster actions. This is not to say that nothing is done to facilitate collaboration, but most park-driven initiatives are implemented with nominal budgets and few dedicated personnel. Consequently, the industrylevel platforms and value networks within parks remain quite weak in comparison to the regional potential. The local science and technology parks have a rather modest role as "real" innovation hubs and do not realize their fullest role and responsibility as true cluster actors.

There is no evidence available to indicate that the Cambridge innovation ecosystem would be very advanced in providing access to pilot, testing, or co-creation plat- forms. The majority of testing and measuring facilities are located within universities and national research institutes, which are equipped and set up primarily for scientific research purposes. Their use produces the highest-quality research results, but their context of use remains relatively closed, especially to SMEs and converging, industry-level technology platforms. In the same way, there are no established living labs within the sub-region and, consequently, no culture for usercentric innovation creation or rapid testing of product/service combinations for emerging markets.

As mentioned earlier, the sub-regional incubation, growth, and globalization services are provided primarily within the university infrastructures, which offer patient and generous support for innovative, entrepreneurial thinking. The St John's Innovation Centre is considered to be the leading provider of comprehensive incubation services in both early and growth stage business development. It implements a full-service model for incubation, collaborates with European incubation partners, and participates in all main regional programs for entrepreneurial training (St John's Innovation Centre, 2010). In this sense, it provides the necessary support structure for the accelerated growth of ambitious, "born global" SMEs and builds up the local venture culture to meet and match the toughest criteria for successful business endeavours. By 2007, St John's had already incubated over 300 high-tech ventures (and helped hundreds of off-site SMEs) and had become a critically important node in many industry-academia networks that characterize the Cambridge Sub-Region.

Now, the regional government and district councils have decided to increase basic investments in sub-regional incubation (and enterprise hub) infrastructures for all leading industry sectors. The latest additions are, for example, Babraham Institute's bioincubator, Allia Future Business Centre, and Ideaspace for pre-start and early-stage ventures (GCP, 2008; Cambridge Network, 2016). These industry-specific incubators operate with principles similar to those of St. John's (albeit on a more modest scale) supporting, on their part, the continuous flows of orchestrated high-tech commercialization in the region. In same context, it has been agreed that all established and new centres and hubs would receive the full support of dedicated intermediary service providers (Judge Business School, Cambridge Enterprise, the Cambridge-MIT Institute, and the Centre for Entrepreneurial Learning, etc.) for their efforts in developing the local venture habitat, business communities, and region-wide value networks. 


\section{Profiling Regional Innovation Ecosystems as Functional Collaborative Systems}

\section{Jukka Viitanen}

The Cambridge Sub-Region is world-renowned for its ability to create and support innovative high-tech startups and growth ventures. The local business landscape comprises an excellent blend of university and corporate spin-offs, SME ventures, and an impressive presence of young, publicly listed companies (professionally supported by numerous technology consultancies, proactive angel investors, and dedicated venture funders). These actors operate in closely interlinked, industry-based value systems and build on mutually beneficial, strategic partnerships, readily transferring information, resources, and technologies for effective (joint) commercialization.

Recent studies (Garnsey \& Heffernan, 2005) estimate that the University of Cambridge alone has created over 300 spin-off and startup companies over the last three decades. Based on financial data collected on 172 of them, they have generated a combined market capitalization of over $£ 5$ billion GBP ( $\$ 8.6$ billion CAD) and employ more than 8,800 people, indicating a significant amount of socio-economic value at both regional and national levels. Concurrently, it is argued that an open attitude towards mutually beneficial technology transfer from academia to industry has had a profound indirect impact on almost all regional business creation. Researchers and scholars have readily placed their global reputation and functional networks at the disposal of smart businesspeople, and boosted venture development to an unprecedented extent. Furthermore, given that as university-based ventures represent only a modest proportion (about $20 \%$ ) of the total venture habitat in the sub-region, it could be argued that the total socio-economic impact of all Cambridge-born SMEs represents a significant proportion of regional wealth and employment.

The ecosystem is particularly good at supporting venture creation and early-stage growth. The resident incubators and innovation centres are usually full of tenants and their clients typically grow fast for the first couple of years while enjoying local support from quality mentors and early-stage financiers. According to recent statistical evidence (Garnsey \& Heffernan, 2005; Library House, 2008), it is clear that the Cambridgeborn ventures are very successful in attracting institutional funding for their early-stage business development. However, the ecosystem-level analysis shows that the strongest SMEs have been equally able to channel significant amounts of equity investments to laterstage growth, and their relative attractiveness in the mergers and acquisitions (M\&A) and initial public offer- ing (IPO) markets of the UK has remained strong the last decades. Overall, the number of publicly quoted companies from the Cambridge industry clusters has increased from one in 1990 to 70 in 2006 (Library House, 2007).

However, the Cambridge innovation ecosystem seems to face continuous challenges in attracting adequate numbers of entrepreneurs and businesses who can tell a compelling story to investors, customers, and the market. Despite the increased professionalism that generations of successful serial entrepreneurs have brought to the cluster, the local venture habitat does not seem to develop parallel to external business environment changes, and it lacks the capacity to transfer the resident innovation processes to meet and match emerging market needs - especially in the service, media, web/Internet, and creative industry sectors (Library House, 2007). The ecosystem is apparently not very good at keeping the venture growth within the sub-region, and often loses its "brightest stars" early-on in their accelerated growth stage. It is notable that, as SMEs grow to a respectable size, they usually domicile abroad and merge with larger entities. In this way, the local venture development and support processes create visible results and wealth, but remain somewhat detached and sidelined from the overall ecosystem development.

As described earlier, the top end of the framework triangle is well-developed and hosts several $R \& D$ centres of globally-renowned industrial giants. In addition to their roles as anchor companies that are actively engaged in local networking, joint R\&D\&I processes, and commercialization of emerging technologies and solutions, it is common for them to seek reciprocal partnerships with first-class research teams, and contract advanced research projects to the resident universities and research institutes. The resident anchor companies are also active partners in the local venture habitat. They often take a leading role in organizing industrywide networks and partnerships for joint innovation creation, channeling a continuous flow of ideas, resources, and technologies to be commercialized in emerging markets. In some cases, they offer ventures an access to their proprietary, global networks, industry-wide distributions systems, and alternative sales channels, accelerating business growth and strengthening the regional value system. At the same time, they are often willing to send their experts and management teams to local networking events, and they encourage their employees to engage in special interest groups and reciprocal learning collaboration activities. In this 


\section{Profiling Regional Innovation Ecosystems as Functional Collaborative Systems} Jukka Viitanen

way, the Cambridge Sub-Regional anchor companies act as business mentors, ecosystem developers, and trusted partners for regional collaboration.

In summary, the Cambridge Sub-Region innovation ecosystem can be characterized as a fully-functional innovation creation platform combining rich regional knowledge assets and collaborative processes in a dynamic public-private partnership context. The sub-region enjoys a true critical mass of first-class businesses and research institutions involved in high-tech $R \& D$ and technology commercialization, generating a dynamic "engine of economic growth" in the broader regional and national contexts. It has turned in an impressive innovation-related performance across a broad range of sectors and is recognized as a key contributor to the UK economy. Consequently, the ecosystem can be seen as regionally important and both nationally and globally relevant with full potential to continue as one of the leading locations for global innovation-creation activities (Figure 4).

Moreover, Cambridge is recognized as one of leading technology clusters in Europe, attracting a continuous flow of talent and funding to support and strengthen its indigenous innovation processes. It readily interconnects local actors under unified horizontal structures, builds a strong chain of "glocal" innovation activities on-site and extends the regional/national value networks gradually to neighbouring regions and innovation hubs. The local hub management structures are actively developed and coordinated by key regional intermediaries, which promotes the open exchange of information, resources, and talent throughout the ecosystem, and facilitates joint actions. However, there are no indications that the local actors seek new opportunities to engage in mutually beneficial partnerships with other top-runner environments or consequent global alliances of trusted innovation partnerships, which may limit ecosystem potential to some extent. Nevertheless, with widespread national appeal and significance due to its positive impact on regional socioeconomic transformations, the Cambridge Sub-Region has been, and continues to be, a model for regional innovation and economic development throughout the world.

\section{Conclusions}

This article presented both a comprehensive theoretical framework and detailed practical evidence of best practices in developing and managing regional innovation ecosystems and hubs. It has been demonstrated that the presented innovation hub framework constitutes a fittingly comprehensive approach to regional innovation ecosystem development, advocating coordinated planning and implementation of the key ecosystem elements and close interplay among the key innovation actors. The framework guides regional planners, political decision makers, and core-hub organizations in addressing ecosystem development from a unified cross-sectoral point of view - as a complete regional master planning challenge, aiming to connect both public and private sector interests for joint innovation action. If managed properly, such collaborative action can lead to mutually reinforcing arrangements for parallel innovation processes, and it can facilitate the efficient distribution of best-practice know-how throughout the ecosystem.

It is argued that the most successful innovation ecosystems of the future will be embedded in a truly globalized, interconnected, and collaborative context, where information, resources, talent, and solutions can flow freely and effectively between mutually complementing or competing locations. Any modern innovation ecosystem must first have a strong basis in the relevant local know-how and pool of talent in order to build on re-

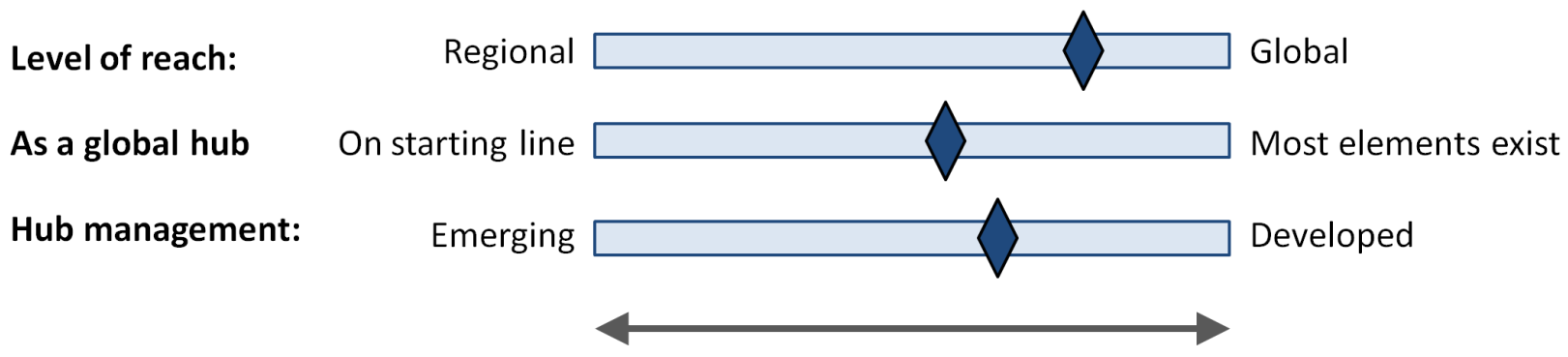

Figure 4. The Cambridge development continuum 


\section{Profiling Regional Innovation Ecosystems as Functional Collaborative Systems} Jukka Viitanen

gional abilities. However, due to changing innovation realities, these locally optimized environments should proactively develop stronger links to complementing networks, preferably together with other equally qualified top-runner ecosystems, to make sure that each individual site would meet the real global targets from day one. In most advanced cases, these interconnected sites could go even one step further and formalize their partnerships as identifiable innovation alliances, which build collaboration on shared, open innovation principles, leveraging trusted relationships for maximum global impact. These alliances could be seen as the ultimate ecosystem generation in collaborative relationship development, serving regional economies as a truly interconnected network of innovation creation platforms and market-entry hubs, providing functional mechanisms for market-specific product and service localizations.

Consequently, the author has begun to advocate for the deliberate adoption of a comprehensive ecosystem development approach to boost real-life regional innovation creation capacity. This approach is built on four principal elements:

1. Grand master planning: As argued above, future development processes for regional innovation ecosystems should be built on comprehensive regional master plans, where all related ecosystem elements could be addressed concurrently to ensure their highest quality, reciprocal compatibility, and relevance in the broader global context. These (top-down) plans translate general collaboration ambitions and ideas into practical development concepts, integrate diverse (bottom-up) innovation-creation practices into manageable entities and introduce comprehensive targets for elevated, ecosystem-level innovation outcomes (joint vision and shared targets).

2. Coordinating service provision: Innovation hub actors can serve ecosystems in several intermediary roles, facilitating cross-industry/domain collaboration and providing professional services in their own specific fields of expertise. They can coordinate the ecosystem-level service provision (use of facilities, development of the KIBS network, upgrades in incubation and growth services, etc.), and safeguard the set quality criteria for planned infrastructure and service structures (audits, evaluations, referrals, etc.). They can guide, promote, and support the other service providers in building up their respective businesses and make sure that all actors strive for top quality and global best practice.
3. Smart orchestration: As identified earlier, coordination of parallel, partly conflicting, sectorial interests, and orchestration of common collaborative interfaces establish one of the most critical management issues for all innovation hub organizations. The public sector actors focus on setting up the policy foundation and related regulatory framework to meet the broadest possible societal needs and actively promote pro-innovation, cross-sectorial collaboration, while the private sector actors plan to line up their inhouse innovation processes for delivering the maximum commercial benefits. As neither side could accomplish their respective missions without the other, they are drawn to establish productive, mutually beneficial partnerships. And, they often look for facilitators to mediate the process. This key orchestration activity is called a smart orchestration, which implies active cross-sectoral communication to reduce overall ambiguity, coupling the sector-specific needs and requirements for a unified ecosystem structure, leading the shared development of the pro-innovation culture and joint processes towards regional ecosystem excellence.

4. Channeling ecosystem resources: It is natural to conclude that the innovation hub actors could also play an important role in channeling and managing the ecosystem-level resource flows to support shared activities and collaborative processes. In most cases, ecosystems can benefit from a professional coordination function, which specializes in core funding issues, application procedures, and channeling resources (both public and private) for effective combinations. Accumulating expertise improves overall process efficiencies and facilitates practical coordination. Consequently, the hub actors could play a key role in advising the other innovation actors on the planning and management of joint infrastructure projects, layer-by-layer development programs, and related coordination of regional innovation creation processes.

In summary, when planning and evaluating innovation ecosystems and hubs, a systemic and comprehensive analysis is not only possible, but if done properly, can reveal the key blueprint for successful ecosystem applications in an increasingly globalizing world. In the course of this study, the author was able to identify gradually emerging, common patterns of behaviour, discover critical subsets of core structural elements for enhanced, joint innovation processes, and unveil the systemic nature of interconnections between actors, actions, and collaborative outcomes. Consequently, it is 


\section{Profiling Regional Innovation Ecosystems as Functional Collaborative Systems} Jukka Viitanen

argued that the related analyses can reveal a formula for replication and speed up the development of the next generation environments - not necessarily directly copying and transferring the core practices "as is" to distant cultural contexts, but rather imitating proven functional behaviour for quality results. This holistic approach to developing future innovation ecosystems and related organizational processes can effectively support local decision makers in achieving the best possible joint innovation outcomes.

\section{About the Author}

Jukka Viitanen is CEO and Managing Partner of Resolute HQ Inc. and former CEO and Partner of Hubconcepts Inc. He has PhD in Information Systems Management and an MSc in Marketing. His academic research has focused on the strategic alignment of business and ICT strategies and the management of global network organizations. Dr. Viitanen has extensive international experience in planning and managing innovation platforms for global excellence. He has served several organizations in Asia and Oceania and managed the Finnish Science Institute in Japan, the Finnish Innovation Center - Finnode Japan, and Asia-Pacific Insight consultancy during his 15 years abroad. Moreover, Dr. Viitanen has developed several governmental programs to facilitate cross-border technology transfer, SME market entry, and S\&T funding collaboration between Finland and its global partners. In his work, he has been responsible for drafting strategies, proposals, and programs for international science and technology collaboration and innovation platform development.

\section{References}

Acs, Z., Arenius, P., Hey, M., \& Minniti, M. 2004. Global Entrepreneurship Monitor 2004: Executive Report. London: London Business School and Babson College.

Autio, E. 2005. GEM - Mazars Special Report on High-Expectation Entrepreneurship. London: London Business School.

Barrell, A. 2005. Changing Culture for Entrepreneurship and Innovation - The Cambridge Story. Paper presented at the Second Annual Conference of the Technopolicy Network, June 16-17, Helsinki, Finland.

Cambridge Network. 2016. Start-Ups and Scale-Ups. Cambridge Network. Accessed December 5, 2016: http://www.cambridgenetwork.co.uk/directories/cambridgecluster/start-ups-and-scale-ups/

Cambridge Technopole. 2008. Cambridge Technopole Report 2008 An Overview of the UK's Leading High-Technology Business Cluster. Cambridge, UK: Cambridge Technopole Group, St John's Innovation Centre Limited.

Cambridgeshire Horizons. 2008. Cambridgeshire Horizons - Driving Forward Sustainable Communities, Business Plan 2008-2011. Cambridge, UK: Cambridgeshire Horizons.

CUG. 2016. Anglia Ruskin University. The Complete University Guide (CUG). Accessed December 5, 2016:

http://www.thecompleteuniversityguide.co.uk/anglia-ruskin/

EEDA. 2009. Innovation Insight - The Innovation Performance of the East of England. Cambridge, UK: Insight East, The Work Foundation, and the East of England Development Agency (EEDA).

Etzkowitz, H. 1997. The Triple Helix: Academy-Industry-Government Relations and the Growth of Neo-Corporatist Industrial Policy in the U.S. In S. Campodall'Orto (Ed.), Managing Technological Knowledge Transfer. EC Social Sciences COST A3: Vol. 4. Brussels: European Commission Directorate General: Science, Research and Development.

European Parliament. 2000. Lisbon European Council 23 and 24 March 2000. Presidency Conclusions. European Parliament. Accessed December 5, 2016: http://www.europarl.europa.eu/summits/lis1_en.htm

Garnsey, E., \& Heffernan, P. 2005. High-Technology Clustering through Spin-Out and Attraction: The Cambridge Case. Regional Studies, 39(8): 1127-1144. http://dx.doi.org/10.1080/00343400500328289

GCP. 2008. Greater Cambridge Sub-Regional Economic Strategy 20092012. Greater Cambridge Partnership, UK.

Granger, J. 2009. Characteristics of Competitive Places: Changing Models for Economic Dynamisms: Cambridge, United Kingdom, In: Proceedings of the IASP World Conference 2009: Future Knowledge Ecosystems - The Opportunity for Science and Technology Parks, Places and Partners. North Carolina, USA.

Greater Cambridge. 2009. Greater Cambridge - Europe's Innovation Capital. Resources and Public Presentation Materials for Greater Cambridge, GCP.

Hautamäki, A. 2008. Kestävä Innovointi: Innovaatiopolitiikka uusien haasteiden edessä. Sitran raportteja 76. Helsinki: Sitra. 


\section{Profiling Regional Innovation Ecosystems as Functional Collaborative Systems}

\section{Jukka Viitanen}

Ishikura, Y. 2006. Global Innovation Ecosystem. In Proceedings of the International Conference on Science and Technology for Sustainability 2006, September 8-9, 2006, Kyoto, Japan.

Kao, J. 2007. Innovation Nation: How America is Losing Its Innovation Edge, Why That Matters and What Can We Do To Get It Back? New York: Free Press.

Launonen, M., \& Viitanen, J. 2011. Hubconcepts: The Global Best Practice for Managing Innovation Ecosystems and Hubs. Helsinki: Hubconcepts Inc.

Laydesdorf, L. 2006. The Triple Helix and Knowledge-Based Innovation Systems. In M. Rebernik et al. (Eds.), Cooperation between the Economic, Academic and Governmental Spheres: Mechanisms and Levers - Proceedings of the 26th Conference on Entrepreneurship and Innovation, March 30-31, 2006, Maribor, Slovenia.

Library House. 2007. Looking Inwards, Reaching Outwards: The Cambridge Cluster Report 2007. Cambridge, UK: The Library House Limited.
Library House. 2008. Competitive Advantage: The Cambridge Cluster Report 2008. Cambridge, UK: The Library House Limited.

Saxenian, A. 2006. The New Argonauts: Regional Advantages in a Global Economy. Cambridge, MA: Harvard University Press.

St John's Innovation Centre. 2010. St John's Innovation Centre. Accessed December 5, 2016: http://www.stjohns.co.uk/

Times Higher Education. 2016. World University Rankings 2016-2017. Times Higher Education. Accessed December 5, 2016: http://www.timeshighereducation.com/world-universityrankings/2017/world-ranking

University of Cambridge. 2010. University of Cambridge. Accessed December 5, 2016:

http://www.cam.ac.uk/

Citation: Viitanen, J. 2016. Profiling Regional Innovation Ecosystems as Functional Collaborative Systems: The Case of Cambridge. Technology Innovation Management Review, 6(12): 6-25. http://timreview.ca/article/1038

Keywords: innovation ecosystem, system thinking, public-private partnership, PPP, orchestration, management, Cambridge 